Article

\title{
Influenza Vaccine Effectiveness Estimates among US Department of Defense Adult Beneficiaries over Four Consecutive Influenza Seasons: A Test-Negative Design Study with Different Control Groups
}

\author{
Wenping $\mathrm{Hu}^{1,2, *}$, Paul A. Sjoberg ${ }^{1,2}$, Laurie S. DeMarcus ${ }^{1,2}$ and Anthony S. Robbins ${ }^{1}$ \\ 1 The Department of Defense Global Emerging Infections Surveillance Branch, Armed Forces Health \\ Surveillance Division, Wright-Patterson Air Force Base, Dayton, OH 45433, USA; \\ paul.sjoberg.1.ctr@us.af.mil (P.A.S.); laurie.demarcus.ctr@us.af.mil (L.S.D.); \\ anthony.robbins.5@us.af.mil (A.S.R.) \\ 2 JYG Innovations LLC, Dayton, OH 45414, USA \\ * Correspondence: wenping.hu.ctr@us.af.mil
}

check for updates

Citation: Hu, W.; Sjoberg, P.A.; DeMarcus, L.S.; Robbins, A.S Influenza Vaccine Effectiveness Estimates among US Department of Defense Adult Beneficiaries over Four Consecutive Influenza Seasons: A Test-Negative Design Study with Different Control Groups. Vaccines 2022, 10, 58. https://doi.org/ $10.3390 /$ vaccines10010058

Academic Editor: Takashi Imai

Received: 30 November 2021

Accepted: 29 December 2021

Published: 31 December 2021

Publisher's Note: MDPI stays neutral with regard to jurisdictional claims in published maps and institutional affiliations.

Copyright: (C) 2021 by the authors. Licensee MDPI, Basel, Switzerland. This article is an open access article distributed under the terms and conditions of the Creative Commons Attribution (CC BY) license (https:// creativecommons.org/licenses/by/ $4.0 /$ )

\begin{abstract}
A test-negative design study with different control groups (influenza test-negative controls, non-influenza virus positive controls, and pan-negative controls) was conducted to assess inactivated influenza vaccine effectiveness (VE) in adults aged $\geq 18$ years, 2016-2017 through 2019-2020 influenza seasons. A database was developed from the US Department of Defense Global Respiratory Pathogen Surveillance Program. VE was estimated using a generalized linear mixed model with logit link and binomial distribution, adjusted for confounding effects. A total of 7114 adults including 2543 medically attended, laboratory-confirmed influenza-positive cases were identified. Using influenza test-negative controls, the adjusted VE in adults was 40\% [95\% confidence interval (CI): 33-46\%] overall, including 46\% (95\% CI: 36-55\%) for influenza A(H1N1)pdm09, 32\% (95\% CI: 19-42\%) for influenza A(H3N2), and 54\% (95\% CI: 44-62\%) for influenza B. The age-stratified analysis showed that VE estimates against influenza A(H1N1)pdm09 (34\%; 95\% CI: -29-66\%) and influenza A(H3N2) (6\%; 95\% CI: $-60-45 \%$ ) were low and non-significant for elderly adults $\geq 65$ years of age. Overall VE estimates against any influenza or by influenza (sub)types in adults were consistent when using influenza test-negative controls, non-influenza virus positive controls, and pan-negative controls. Inactivated influenza vaccination provided moderate protection against influenza virus infection, based on the analysis from a large number of adults aged $\geq 18$ years over multiple influenza seasons.
\end{abstract}

Keywords: influenza; inactivated influenza vaccine; vaccine effectiveness; test-negative design; adult

\section{Introduction}

Seasonal influenza primarily causes human respiratory disease in all ages. Specifically, elderly adults ( $\geq 65$ years of age) are at high risk of influenza virus infection and severe influenza-related complications including hospitalization and death [1]. Influenza vaccination is considered the most effective measure available to protect against influenza viruses, combat influenza virus infection and lessen disease severity. Every year, the Department of Defense (DoD) Global Respiratory Pathogen Surveillance (DoDGRS) Program performs routine respiratory pathogen surveillance among DoD service members and their beneficiaries, allowing annual estimates of influenza vaccine effectiveness (VE) [2-5]. Annual estimates of VE are necessary as the circulating influenza viruses differ from year to year. Nevertheless, due to the small sample size, it might not be possible to accurately estimate $\mathrm{VE}$ in some age-stratified groups (e.g., elderly adults aged $\geq 65$ years) in a single influenza season. There was a need thus to conduct VE analyses with greater statistical power, by combining all available data over multiple influenza seasons. 
The test-negative design has been widely used in influenza VE studies since 2005 [6]. In this design, patients with influenza-like illness symptoms who tested influenza negative are commonly treated as controls. The influenza negative controls include patients who tested for other respiratory virus positive (non-influenza virus positive) and those tested for other respiratory virus negative [panel (pan)-negative]. Alternatively, the non-influenza virus positive or the pan-negative patients could be chosen as controls in a test-negative design study. In case-control studies, one of important assumptions is that control selection should be independent of exposure [7]. Nevertheless, due to potential virus interference [8], the risk of infection by a non-influenza virus could be influenced by influenza vaccination. As a result, this assumption may be violated in influenza test-negative design studies, which leads to biased control selection, consequently affecting valid VE estimates. Previous studies $[6,9,10]$ have examined the impact of selection of different control groups on VE estimates; but the findings in their studies have been inconsistent. Feng et al. [7] conducted a meta-analysis to estimate influenza VE in the test-negative design study using alternative control groups, and found no differences in VE estimates based on the choice of control group. However, it should be noted that the conclusion drawn from the meta-analysis study [7] was based on limited 12 test-negative design studies; Moreover, there were vast differences in the age group, outpatient/inpatient settings, and countries investigated among the studies. Such variations among the studies may limit the interpretation of the meta-analysis results. Therefore, further research would undoubtedly provide more evidence to help determine right choice of control group in influenza VE studies.

Analyses reported herein are based on DoDGRS data over four consecutive influenza seasons. One objective of this study was to evaluate the effectiveness of inactivated influenza vaccine against medically attended, laboratory-confirmed influenza in adults aged $\geq 18$ years among DoD beneficiaries in the United States seeking outpatient care over four influenza seasons (2016-2017 through 2019-2020). A secondary objective was to compare VE estimates using influenza test-negative controls to those using two other control groups (i.e., non-influenza virus positive controls and pan-negative controls) in the same study population.

\section{Methods}

\subsection{Study Population}

We used DoDGRS data over four influenza seasons from 2016-2017 to 2019-2020. All patients seeking outpatient medical care for influenza-like illness (ILI) clinical conditions were selected. ILI was defined if the patient has (1) a fever greater than or equal to $38{ }^{\circ} \mathrm{C}$ and a cough or sore throat which presents within $72 \mathrm{~h}$ after illness onset, or (2) physician determined ILI. Respiratory specimens were collected from ILI patients by nasopharyngeal wash or nasopharyngeal swab, and subject to testing via a multiplex respiratory pathogen panel, reverse transcription polymerase chain reaction (RT-PCR) and/or viral culture. Thus, the influenza viruses and other respiratory pathogens were identified and confirmed. Influenza virus type and subtype/lineage were identified when the respiratory specimen tested positive for influenza virus. Patients who had received at least one inactivated influenza vaccine dose 14 days or more before the onset of an ILI, were considered vaccinated. Patients who otherwise had not received vaccination before the onset of an ILI, were considered unvaccinated. Vaccination status was verified through the records from the DoD Electronic Immunization Tracking System or self-reported questionnaire for each patient. Patients with an unknown vaccination status or type, or vaccinated $<14$ days prior to the onset of an ILI were excluded. Vaccine type in the present study were limited to standard-dose influenza vaccine only; thus, patients who received influenza vaccine other than standard-dose influenza vaccine were excluded.

The patients in the present study were limited to adult beneficiaries from the DoDGRS program sentinel or participating sites in the United States. 


\subsection{Age Stratification}

Age was classified into three groups (18-49 years, 50-64 years, and $\geq 65$ years). The rationale for age classification was based on the fact that adults 65 years of age or older are at higher risk for influenza virus infection and severe influenza-related complications [1]. Moreover, when influenza vaccine supply is limited, vaccination efforts should focus on specific populations including all persons aged $\geq 50$ years [11]. It was of interest to evaluate VE against influenza viruses in adults younger than 65 years of age with two further stratified age categories (18-49 years and 50-64 years).

\subsection{Statistical Analysis}

Prior to combining data over four influenza seasons, the range of surveillance weeks in each influenza season was limited to November to April of the following year for the VE analysis, when approximately $10 \%$ or greater influenza positivity rate occurred, with an aim to minimize any potential bias due to a high ratio of influenza negative to influenza positive that would typically occur earlier or later in the influenza season.

All data was combined by influenza season, and analysis was performed using generalized linear mixed models with logit link and binomial distribution. Influenza season was treated as a random effect in the model. The odds of influenza vaccination among adults with laboratory-confirmed influenza positive (cases) were compared to the odds of influenza vaccination among adults who were tested influenza negative (controls), or to the odds of influenza vaccination among non-influenza virus positive controls or pan-negative controls. VE was calculated as $(1-$ adjusted odds ratio $) \times 100 \%$. All potential confounding factors, such as age, gender, specimen collection date, or geographical region were initially evaluated. Only those factors that changed the crude odds ratio by $\geq 5 \%$ were included in the generalized linear mixed models to adjust VE. In addition to overall VE estimated against any influenza viruses in the entire adult population, we estimated VE by influenza virus (sub)type in separate models [i.e., influenza $\mathrm{A}(\mathrm{H} 1 \mathrm{~N} 1) \mathrm{pdm} 09$, influenza $\mathrm{A}(\mathrm{H} 3 \mathrm{~N} 2)$, or influenza B], and in stratified models by age category. The point estimate of VE was considered statistically significant when the lower limit of the associated $95 \%$ confidence interval (CI) did not contain zero or a negative value.

Influenza vaccination coverages were compared between two other control groups (non-influenza virus positive controls vs. pan-negative controls) using the generalized linear mixed models, adjusted for all the confounders identified in the influenza VE analysis. A $p<0.05$ was considered statistically significant. All analyses were conducted using SAS Enterprise Guide 7.1 (SAS Institute Inc., Cary, NC, USA).

\section{Results}

\subsection{Patient Characteristics}

The characteristics of the study population over four influenza seasons (2016-2017 to 2019-2020) is shown in Table 1. During the four influenza seasons, a total of 7114 adults were identified for the VE analysis; among whom there were $3573(50.2 \%)$ adults aged 18-49 years, $2230(31.4 \%)$ adults aged 50-64 years, and $1311(18.4 \%)$ adults aged $\geq 65$ (Table 1). Among these adults, $4071(57.2 \%)$ were influenza vaccinated and 3043 $(42.8 \%)$ were influenza unvaccinated. Vaccination coverage increased from $47.9 \%$ at age of $18-49$ years to $58.6 \%$ at age of $50-64$ years, reached as high as $80.3 \%$ at age of $\geq 65$ years. Of the total 2543 influenza-positive cases, influenza A(H1N1)pdm09, influenza A(H3N2), and influenza B were $793(31.2 \%), 909$ (35.7\%), and $590(23.2 \%)$, respectively, with the remaining being $248(9.8 \%)$ non-subtyped influenza A and $3(0.1 \%)$ influenza co-infection (Table 1). Among all 4571 patients testing negative for influenza virus, $1250(27.3 \%)$ tested positive for non-influenza virus and $3321(72.7 \%)$ tested negative for both influenza and other respiratory viruses. The most frequently detected non-influenza viruses causing viral infection were rhinovirus/enterovirus $(422 ; 33.8 \%)$, seasonal coronavirus $(329 ; 26.3 \%)$, followed by human metapneumovirus $(218 ; 17.4 \%)$. 
Table 1. Characteristics of study population used for vaccine effectiveness analysis over four influenza seasons (2016-2017 to 2019-2020).

\begin{tabular}{|c|c|c|c|c|c|}
\hline & Overall & 2016-2017 & 2017-2018 & 2018-2019 & 2019-2020 \\
\hline Characteristic & $\mathbf{N}(\%)$ & $\mathbf{N}(\%)$ & N (\%) & $\mathbf{N}(\%)$ & $\mathbf{N}(\%)$ \\
\hline \multicolumn{6}{|l|}{ Gender } \\
\hline Male & $2360(33.17)$ & $225(28.96)$ & $551(32.58)$ & 865 (36.12) & $719(31.94)$ \\
\hline Female & $4754(66.83)$ & $552(71.04)$ & $1140(67.42)$ & $1530(63.88)$ & $1532(68.06)$ \\
\hline \multicolumn{6}{|l|}{ Age } \\
\hline $18-49$ years & $3573(50.22)$ & $471(60.62)$ & $828(48.97)$ & $1058(44.18)$ & $1216(54.02)$ \\
\hline 50-64 years & 2230 (31.35) & 249 (32.05) & $566(33.47)$ & $706(29.48)$ & 709 (31.5) \\
\hline$\geq 65$ years & $1311(18.43)$ & $57(7.34)$ & $297(17.56)$ & $631(26.35)$ & $326(14.48)$ \\
\hline \multicolumn{6}{|l|}{ Month of illness } \\
\hline November & $231(3.25)$ & $14(1.8)$ & $53(3.13)$ & $0(0)$ & $164(7.29)$ \\
\hline December & $975(13.71)$ & $120(15.44)$ & $246(14.55)$ & $272(11.36)$ & 337 (14.97) \\
\hline January & $1925(27.06)$ & $170(21.88)$ & $544(32.17)$ & $587(24.51)$ & $624(27.72)$ \\
\hline February & $2009(28.24)$ & $220(28.31)$ & $484(28.62)$ & $721(30.1)$ & $584(25.94)$ \\
\hline March & 1575 (22.14) & $174(22.39)$ & $247(14.61)$ & $612(25.55)$ & $542(24.08)$ \\
\hline April & $399(5.61)$ & 79 (10.17) & $117(6.92)$ & $203(8.48)$ & $0(0)$ \\
\hline \multicolumn{6}{|l|}{$\begin{array}{l}\text { Geographic } \\
\text { region a }\end{array}$} \\
\hline Region 1 & $23(0.32)$ & $2(0.26)$ & $2(0.12)$ & $2(0.08)$ & $17(0.76)$ \\
\hline Region 2 & $479(6.73)$ & $90(11.58)$ & $182(10.76)$ & $115(4.8)$ & $92(4.09)$ \\
\hline Region 3 & $489(6.87)$ & $87(11.2)$ & $177(10.47)$ & $59(2.46)$ & $166(7.37)$ \\
\hline Region 4 & $931(13.09)$ & $129(16.6)$ & $302(17.86)$ & $236(9.85)$ & 264 (11.73) \\
\hline Region 5 & $996(14)$ & $18(2.32)$ & $363(21.47)$ & $264(11.02)$ & 351 (15.59) \\
\hline Region 6 & $2554(35.9)$ & $167(21.49)$ & $305(18.04)$ & $1389(58)$ & $693(30.79)$ \\
\hline Region 7 & $246(3.46)$ & $54(6.95)$ & $57(3.37)$ & $29(1.21)$ & $106(4.71)$ \\
\hline Region 8 & $682(9.59)$ & $90(11.58)$ & $172(10.17)$ & $157(6.56)$ & $263(11.68)$ \\
\hline Region 9 & $471(6.62)$ & $76(9.78)$ & $97(5.74)$ & $66(2.76)$ & $232(10.31)$ \\
\hline Region 10 & $243(3.42)$ & $64(8.24)$ & $34(2.01)$ & $78(3.26)$ & $67(2.98)$ \\
\hline \multicolumn{6}{|l|}{ Vaccine status } \\
\hline Vaccinated & $4071(57.23)$ & $330(42.47)$ & 841 (49.73) & $1596(66.64)$ & 1304 (57.93) \\
\hline Unvaccinated & 3043 (42.77) & $447(57.53)$ & $850(50.27)$ & 799 (33.36) & 947 (42.07) \\
\hline \multicolumn{6}{|l|}{ Influenza } \\
\hline $\mathrm{A}(\mathrm{H} 1 \mathrm{~N} 1) \mathrm{pdm} 09$ & 793 (11.15) & $4(0.51)$ & $99(5.85)$ & $185(7.72)$ & $505(22.43)$ \\
\hline $\mathrm{A}(\mathrm{H} 3 \mathrm{~N} 2)$ & 909 (12.78) & $264(33.98)$ & $419(24.78)$ & $208(8.68)$ & $18(0.8)$ \\
\hline A/not subtyped & $248(3.49)$ & $0(0)$ & $0(0)$ & $242(10.1)$ & $6(0.27)$ \\
\hline B & $590(8.29)$ & $78(10.04)$ & $257(15.2)$ & $18(0.75)$ & $237(10.53)$ \\
\hline Dual influenza & $3(0.04)$ & $0(0)$ & $3(0.18)$ & $0(0)$ & $0(0)$ \\
\hline Non-influenza & $4571(64.25)$ & $431(55.47)$ & $913(53.99)$ & $1742(72.73)$ & $1485(65.97)$ \\
\hline
\end{tabular}

a The U.S. Health and Human Services Regions 1-10, except for Guam, Alaska, and Hawaii.

When influenza unvaccinated, there were fewer patients ( $\geq 18$ years of age) in noninfluenza virus positive controls than in pan-negative controls (483 vs. 1229); likewise, fewer patients were observed (767 vs. 2092) when influenza vaccinated. Additionally, regardless of vaccination status in different age groups, there were fewer patients in non-influenza virus positive controls versus pan-negative controls (Table 2). 
Table 2. Characteristics of patients by vaccination status in the test-negative design study with different control groups.

\begin{tabular}{|c|c|c|c|c|c|c|c|c|c|c|c|c|c|c|}
\hline & \multicolumn{7}{|c|}{ Unvaccinated } & \multicolumn{7}{|c|}{ Vaccinated } \\
\hline & \multirow[b]{3}{*}{ Case } & \multicolumn{6}{|c|}{ Control Groups } & \multirow[b]{3}{*}{ Case } & \multicolumn{6}{|c|}{ Control Groups } \\
\hline & & \multicolumn{2}{|c|}{$\begin{array}{c}\text { Influenza } \\
\text { Test-Negative }\end{array}$} & \multicolumn{2}{|c|}{$\begin{array}{l}\text { Non-Influenza } \\
\text { Virus Positive }\end{array}$} & \multicolumn{2}{|c|}{ Pan-Negative } & & \multicolumn{2}{|c|}{$\begin{array}{c}\text { Influenza } \\
\text { Test-Negative }\end{array}$} & \multicolumn{2}{|c|}{$\begin{array}{l}\text { Non-Influenza } \\
\text { Virus Positive }\end{array}$} & \multicolumn{2}{|c|}{ Pan-Negative } \\
\hline & & Control & $\begin{array}{l}\text { Case } \\
(\%)\end{array}$ & Control & $\begin{array}{l}\text { Case } \\
(\%)\end{array}$ & Control & $\begin{array}{l}\text { Case } \\
(\%)\end{array}$ & & Control & $\begin{array}{l}\text { Case } \\
(\%)\end{array}$ & Control & $\begin{array}{l}\text { Case } \\
(\%)\end{array}$ & Control & $\begin{array}{l}\text { Case } \\
(\%)\end{array}$ \\
\hline 18-49 years & & & & & & & & & & & & & & \\
\hline $\begin{array}{c}\text { Influenza } \\
\text { A(H1N1)pdm09 }\end{array}$ & 227 & 1068 & 17.53 & 297 & 43.32 & 771 & 22.75 & 184 & 1189 & 13.40 & 363 & 33.64 & 826 & 18.22 \\
\hline $\begin{array}{l}\text { Influenza } \\
\text { A(H3N2) }\end{array}$ & 292 & 1068 & 21.47 & 297 & 49.58 & 771 & 27.47 & 169 & 1189 & 12.44 & 363 & 31.77 & 826 & 16.98 \\
\hline Influenza B & 217 & 1068 & 16.89 & 297 & 42.22 & 771 & 21.96 & 111 & 1189 & 8.54 & 363 & 23.42 & 826 & 11.85 \\
\hline Any influenza ${ }^{a}$ & 794 & 1068 & 42.64 & 297 & 72.78 & 771 & 50.73 & 522 & 1189 & 30.51 & 363 & 58.98 & 826 & 38.72 \\
\hline $\begin{array}{c}\text { 50-64 years } \\
\text { Influenza } \\
\text { A(H1N1)pdm09 }\end{array}$ & 160 & 473 & 25.28 & 148 & 51.95 & 325 & 32.99 & 153 & 863 & 15.06 & 232 & 39.74 & 631 & 19.52 \\
\hline $\begin{array}{l}\text { Influenza } \\
\text { A(H3N2) }\end{array}$ & 148 & 473 & 23.83 & 148 & 50.00 & 325 & 31.29 & 158 & 863 & 15.48 & 232 & 40.51 & 631 & 20.03 \\
\hline Influenza B & 122 & 473 & 20.50 & 148 & 45.19 & 325 & 27.29 & 85 & 863 & 8.97 & 232 & 26.81 & 631 & 11.87 \\
\hline Any influenza ${ }^{a}$ & 450 & 473 & 48.75 & 148 & 75.25 & 325 & 58.06 & 444 & 863 & 33.97 & 232 & 65.68 & 631 & 41.30 \\
\hline $\begin{array}{c}\geq 65 \text { years } \\
\text { Influenza } \\
\text { A(H1N1)pdm09 }\end{array}$ & 19 & 171 & 10.00 & 38 & 33.33 & 133 & 12.50 & 50 & 807 & 5.83 & 172 & 22.52 & 635 & 7.30 \\
\hline $\begin{array}{l}\text { Influenza } \\
\text { A(H3N2) }\end{array}$ & 35 & 171 & 16.99 & 38 & 47.95 & 133 & 20.83 & 107 & 807 & 11.71 & 172 & 38.35 & 635 & 14.42 \\
\hline Influenza B & 21 & 171 & 10.94 & 38 & 35.59 & 133 & 13.64 & 34 & 807 & 4.04 & 172 & 16.50 & 635 & 5.08 \\
\hline Any influenza ${ }^{a}$ & 87 & 171 & 33.72 & 38 & 69.60 & 133 & 39.55 & 246 & 807 & 23.36 & 172 & 58.85 & 635 & 27.92 \\
\hline $\begin{array}{c}\geq 18 \text { years } \\
\text { Influenza } \\
\text { A(H1N1)pdm09 }\end{array}$ & 406 & 1712 & 19.17 & 483 & 45.67 & 1229 & 24.83 & 387 & 2859 & 11.92 & 767 & 33.54 & 2092 & 15.61 \\
\hline $\begin{array}{l}\text { Influenza } \\
\text { A(H3N2) }\end{array}$ & 475 & 1712 & 21.72 & 483 & 49.58 & 1229 & 27.88 & 434 & 2859 & 13.18 & 767 & 36.14 & 2092 & 17.18 \\
\hline Influenza B & 360 & 1712 & 17.37 & 483 & 42.70 & 1229 & 22.66 & 230 & 2859 & 7.45 & 767 & 23.07 & 2092 & 9.91 \\
\hline Any influenza ${ }^{a}$ & 1331 & 1712 & 43.74 & 483 & 73.37 & 1229 & 51.99 & 1212 & 2859 & 29.77 & 767 & 61.24 & 2092 & 36.68 \\
\hline
\end{tabular}

${ }^{a}$ Including influenza A(H1N1)pdm09, influenza A(H3N2), influenza B, influenza A/not subtyped, and influenza co-infection.

\subsection{Confounding Factors Assessment}

Among all potential confounders examined, month of specimen collected (i.e., onemonth period from November to April of the following year), geographic region of specimen collected (i.e., the U.S. Health and Human Services Regions 1-10), and age groups (i.e., $18-49$ years, $50-64$ years, and $\geq 65$ years) were the variables that changed the crude odds ratio by $\geq 5 \%$. Therefore, month of specimen collected, region, and age group were included in the models to estimate overall VE and the VE stratified by influenza type/subtypes or age groups. Moreover, the same set of confounding factors were used in all models to estimate the influenza VE when using different control groups.

\subsection{Overall $V E$}

Using influenza test-negative controls, adjusted VE against laboratory-confirmed influenza for medically attended adults, was $40 \%$ (95\% CI: 33-46\%) overall, including $46 \%$ (95\% CI: 36-55\%) against influenza A(H1N1)pdm09, 32\% (95\% CI: 19-42\%) against influenza A(H3N2), and 54\% (95\% CI: 44-62\%) against influenza B (Table 3). When controls were restricted to patients who tested non-influenza virus positive the adjusted VE was $44 \%$ (95\% CI: 35-52\%) overall, including 50\% (95\% CI: 38-59\%) against influenza A(H1N1)pdm09, 35\% (95\% CI: 19-48\%) against influenza A(H3N2), and 57\% (95\% CI: 46-66\%) against influenza B (Table 3). In addition, when controls were restricted to patients who tested pan-negative, the adjusted VE was 38\% (95\% CI: 30-45\%) overall, including 
45\% (95\% CI: 33-54\%) against influenza A(H1N1)pdm09, 28\% (95\% CI: 14-40\%) against influenza A(H3N2), and 52\% (95\% CI: 41-61\%) against influenza B (Table 3).

Table 3. Adjusted vaccine effectiveness in adults aged $\geq 18$ years.

\begin{tabular}{|c|c|c|c|c|c|c|}
\hline & \multicolumn{6}{|c|}{ Controls } \\
\hline & \multicolumn{2}{|c|}{$\begin{array}{c}\text { Influenza } \\
\text { Test-Negative }\end{array}$} & \multicolumn{2}{|c|}{$\begin{array}{c}\text { Non-Influenza Virus } \\
\text { Positive }\end{array}$} & \multicolumn{2}{|c|}{ Pan-Negative } \\
\hline & $\operatorname{VE~}(\%)^{a}$ & $\begin{array}{c}95 \% \text { CI } \\
(\%)\end{array}$ & $\operatorname{VE~(\% )}{ }^{a}$ & $\begin{array}{c}95 \% \text { CI } \\
(\%)\end{array}$ & $\operatorname{VE~(\% )}{ }^{a}$ & $\begin{array}{c}95 \% \mathrm{CI} \\
(\%)\end{array}$ \\
\hline \multicolumn{7}{|l|}{ 18-49 years } \\
\hline $\begin{array}{l}\text { Influenza } \\
\mathrm{A}(\mathrm{H} 1 \mathrm{~N} 1) \mathrm{pdm} 09\end{array}$ & 41 & $25-53$ & 52 & $36-64$ & 35 & $16-49$ \\
\hline $\begin{array}{l}\text { Influenza } \\
\text { A(H3N2) }\end{array}$ & 42 & $26-54$ & 51 & $34-64$ & 36 & $18-50$ \\
\hline Influenza B & 55 & $41-65$ & 62 & $48-72$ & 51 & $35-63$ \\
\hline $\begin{array}{c}\text { Any } \\
\text { influenza b }\end{array}$ & 44 & $35-52$ & 53 & $42-62$ & 39 & $28-48$ \\
\hline \multicolumn{7}{|l|}{ 50-64 years } \\
\hline $\begin{array}{l}\text { Influenza } \\
\text { A(H1N1)pdm09 }\end{array}$ & 53 & $38-65$ & 47 & $24-62$ & 57 & $41-68$ \\
\hline $\begin{array}{l}\text { Influenza } \\
\text { A(H3N2) }\end{array}$ & 26 & $1-45$ & 13 & $-30-41$ & 26 & $-1-46$ \\
\hline Influenza B & 56 & $38-68$ & 52 & $29-68$ & 57 & $39-70$ \\
\hline $\begin{array}{c}\text { Any } \\
\text { influenza b }\end{array}$ & 41 & $29-51$ & 36 & $16-51$ & 43 & $30-53$ \\
\hline \multicolumn{7}{|l|}{$\geq 65$ years } \\
\hline $\begin{array}{l}\text { Influenza } \\
\mathrm{A}(\mathrm{H} 1 \mathrm{~N} 1) \mathrm{pdm} 09\end{array}$ & 34 & $-29-66$ & 41 & $-26-73$ & 27 & $-46-63$ \\
\hline $\begin{array}{l}\text { Influenza } \\
\text { A(H3N2) }\end{array}$ & 6 & $-60-45$ & 15 & $-82-60$ & -4 & $-84-41$ \\
\hline Influenza B & 60 & $19-80$ & 65 & $18-85$ & 55 & $6-78$ \\
\hline $\begin{array}{c}\text { Any } \\
\text { influenza }\end{array}$ & 28 & $0-49$ & 40 & $0-64$ & 24 & $-8-47$ \\
\hline \multicolumn{7}{|l|}{$\geq 18$ years } \\
\hline $\begin{array}{l}\text { Influenza } \\
\mathrm{A}(\mathrm{H} 1 \mathrm{~N} 1) \mathrm{pdm} 09\end{array}$ & 46 & $36-55$ & 50 & $38-59$ & 45 & $33-54$ \\
\hline $\begin{array}{l}\text { Influenza } \\
\text { A(H3N2) }\end{array}$ & 32 & $19-42$ & 35 & $19-48$ & 28 & $14-40$ \\
\hline Influenza B & 54 & $44-62$ & 57 & $46-66$ & 52 & $41-61$ \\
\hline $\begin{array}{c}\text { Any } \\
\text { influenza b }\end{array}$ & 40 & $33-46$ & 44 & $35-52$ & 38 & $30-45$ \\
\hline
\end{tabular}

\subsection{VE by Age Groups}

Using influenza test-negative controls, the adjusted VE estimates against any influenza were $44 \%$ (95\% CI: $35-52 \%$ ) in adults aged $18-49$ years, and $41 \%$ (95\% CI: $29-51 \%$ ) in adults aged 50-64 years, then declined to $28 \%$ (95\% CI: $0-49 \%$ ) in adults aged $\geq 65$ years. In comparison with those VE using influenza test-negative controls, the VE estimates against any influenza in different age groups were consistent when using pan-negative controls (Table 3). On the other hand, when using non-influenza virus positive controls, the adjusted VE against any influenza was higher (53\%; 95\% CI: 42-62\%) in adults aged $18-49$ years, compared to the VE estimates in adults aged 50-64 years (36\%; $95 \%$ CI: $16-51 \%$ ) or in adults aged $\geq 65$ years ( $40 \% ; 95 \%$ CI: $0-64 \%$ ).

Regardless of different control groups used, the adjusted VE estimates against influenza A(H1N1)pdm09 were higher in younger adults (18-49 years and 50-64 years of 
age), compared to those in adults aged $\geq 65$ years. The adjusted VE against influenza $\mathrm{A}(\mathrm{H} 3 \mathrm{~N} 2)$ was similar to that against influenza A(H1N1)pdm09 in adults aged 18-49 years; but as age increased, the VE greatly decreased. In contrast, the VE estimates against influenza B exceeded $50 \%$ in all age groups, but a wider $95 \% \mathrm{CI}$ of the VE occurred in adults aged $\geq 65$ years (Table 3 ).

\subsection{Influenza Vaccination Coverage}

There was no difference in vaccination coverage between non-influenza virus positive controls (61.4\%) and pan-negative controls (63.0\%) in adults (Table 4). By age groups, vaccination coverage was higher ( 55.0 vs. $51.7 \% ; p=0.012$ ) in adults aged $18-49$ years for non-influenza virus positive controls vs. pan-negative controls; however, no statistically different vaccine coverages were found in adults aged 50-64 years (61.1 vs. 66.0\%) and in adults aged $\geq 65$ years ( 61.4 vs. $63.0 \%$ ) between these two control groups.

Table 4. Influenza vaccine coverage among patients tested non-influenza virus positive and patients tested pan-negative.

\begin{tabular}{cccccc}
\hline & \multicolumn{2}{c}{$\begin{array}{c}\text { Non-Influenza Virus } \\
\text { Positive Controls }\end{array}$} & \multicolumn{2}{c}{ Pan-Negative Controls } & \multirow{2}{*}{$\boldsymbol{p}$-Value } \\
\cline { 2 - 5 } & Total & Vaccinated $\mathbf{( \% )}$ & Total & Vaccinated (\%) & \\
\hline 18-49 years & 660 & $363(55.0)$ & 1597 & $826(51.7)$ & 0.012 \\
50-64 years & 380 & $232(61.1)$ & 956 & $631(66.0)$ & 0.198 \\
$\geq 65$ years & 210 & $172(81.9)$ & 768 & $635(82.7)$ & 0.709 \\
$\geq 18$ years & 1250 & $767(61.4)$ & 3321 & $2092(63.0)$ & 0.245 \\
\hline
\end{tabular}

\section{Discussion}

We report the pooled estimates of VE against medically attended, laboratory-confirmed influenza in adults over the 2016-2020 influenza seasons (Table 3). As analyzed using influenza test-negative controls, significant protection of standard-dose influenza vaccine against any influenza was found in adults $(40 \%)$, while by influenza (sub)type, the protection was higher against influenza $\mathrm{A}(\mathrm{H} 1 \mathrm{~N} 1)$ pdm09 and influenza $\mathrm{B}$, but lower against influenza A(H3N2). Moreover, the age-stratified analysis indicated the overall VE against any influenza viruses was lower in adults aged $\geq 65$ years compared with younger adults (18-49 years or 50-64 years of age).

Previously, we conducted the VE analysis in children aged 6 months-17 years that included DoDGRS data in outpatient settings within the same seasons [12]. It was shown that the VE against influenza in children was 42\% (95\% CI: 37-47\%) overall, including 55\% (95\% CI: 47-61\%) against influenza A(H1N1)pdm09, 37\% (95\% CI: 28-45\%) against influenza A(H3N2), and 49\% (95\% CI: 41-55\%) against influenza B. In comparison, influenza vaccine offered similar protections in adults overall and against influenza $B$, and lower but comparable protections against influenza A(H1N1)pdm09 and influenza A(H3N2).

By age groups, Belongia et al. [13] found in their meta-analysis of test-negative design studies from 2004-2015, that the pooled VE against medically attended influenza in working age adults (20-64 years old) and older adults (>60 years) were 73\% (95\% CI: 52-84\%) and 62\% (95\% CI: 36-78\%) for influenza A(H1N1)pdm09, 35\% (95\% CI: 14-51\%) and $24 \%$ (95\% CI: $-6-45 \%$ ) for influenza A(H3N2), and 54\% (95\% CI: $16-75 \%)$ and $63 \%$ (95\% CI: 33-79\%) for influenza B, respectively. More recently, Russell et al. [14] combined data among outpatients aged $\geq 18$ years from the US Flu VE Network over five influenza seasons (2011-2012 through 2015-2016). Their analysis showed that the adjusted VE in adults aged $18-49$ years and $\geq 65$ years were $48 \%$ (95\% CI: $38-57 \%$ ) and $49 \%$ (95\% CI: 22-66\%) for influenza A(H1N1)pdm09, 21\% (95\% CI: 10-32\%) and 14\% (95\% CI: - 14-36\%) for influenza A(H3N2), and 55\% (95\% CI: 45-63\%) and 62\% (95\% CI: 44-74\%) for influenza $B$, respectively. Compared to the findings from these studies $[13,14]$, we found similar VE estimates against influenza B in adults with different ages. Moreover, lower VE estimate 
against influenza $\mathrm{A}(\mathrm{H} 3 \mathrm{~N} 2)$ was consistently observed in adults aged $\geq 65$ years than in adults aged 18-49 years. However, in the present study, the VE estimate against influenza $\mathrm{A}(\mathrm{H} 1 \mathrm{N1}$ )pdm09 in adults aged $\geq 65$ years was lower and non-significant. Recent studies in the US [15-17], which performed VE analysis each season individually over the three seasons from 2016-2017 to 2018-2019, have also shown low and non-significant effectiveness of influenza vaccination against influenza A(N1H1)pdm09 and influenza A(H3N2) in adults aged $\geq 65$ years, with the exception of no assessment of VE against influenza A(H1N1)pdm09 being reported during 2016-2017 influenza season [15].

Our findings clearly demonstrated inactivated influenza vaccine was less effective against influenza $\mathrm{A}(\mathrm{H} 1 \mathrm{~N} 1)$ pdm09 and influenza $\mathrm{A}(\mathrm{H} 3 \mathrm{~N} 2)$ in adults aged $\geq 65$ years. Age is one of the major host factors (e.g., age, history of infection and prior vaccination, health status, etc.) to determine influenza vaccine response [18]. The decreased VE in adults aged $\geq 65$ years is usually attributed to immunosenescence, a gradual decline in immune function as age advances $[19,20]$. Indeed, the decline of immune function in older people would lead to greater susceptibility to influenza virus and affect their ability to respond to influenza vaccination. The protection provided by influenza vaccine is based on induction of antibodies. Goodwin et al. [21] showed that antibody response to influenza vaccine in the elderly is considerably lower than in younger adults. In addition, there is increasing evidence suggesting that intra-season waning of influenza vaccine protection occurs [22,23], and such waning immunity is more pronounced for older adults [24].

Several studies have suggested that repeated vaccination would impact vaccine protection against influenza virus, which might consequently alter VE estimates [25,26]. The underlying immunologic mechanisms for potential vaccine interference are not well understood [27]. Khurana et al. [28] observed a significant negative impact of repeat vaccination on antibody affinity maturation, which may contribute to lower VE of influenza vaccine. Further, age may confound the effect of prior vaccination history on the response to influenza vaccination [29]. Annual influenza vaccination is recommended for specific populations at high risk, including older adults [11]. Older people may thus have more opportunities to receive repeated influenza vaccination. The highest influenza vaccination coverage was evident in adults aged $\geq 65$ years $(80.3 \%)$ in the present study, which is consistent with the previous findings in the US study [30]. Therefore, greater potential impact of repeated vaccination on VE might be expected in adults aged $\geq 65$ years, compared to younger adults.

The impact of three control groups on VE estimates in adults has been evaluated in the present study. Overall VE or VE by influenza (sub)types estimated using non-influenza virus positive controls or pan-negative controls were similar, or comparable to those estimated using test-negative controls. Additionally, the $95 \%$ CI of VE estimates widely overlapped (Table 3). Nevertheless, VE estimates by age groups presented some variations based on the three control groups used. We found that the influenza test-negative and the pan-negative control groups gave more consistent VE estimates (Table 3). It appeared that VE estimates using non-influenza virus positive controls were higher than VE estimates using influenza test-negative controls or pan-negative controls in adults aged 18-49 years and in all adults, but lower in adults aged 50-64 years. van Doorn et al. [10] found in their study conducted in the Dutch population from 2003 to 2014, that influenza estimates in both the main and subgroup analyses using non-influenza virus positive controls were the highest among three different control groups used. Additionally, highest VE estimate using non-influenza virus positive controls was observed in an earlier study [31]. In contrast, Feng et al. [6] estimated the VE over three influenza seasons (2010-2013) in outpatient settings, and observed that VE estimates using each of the three control groups were consistent overall or when stratified by age groups. In the study of Pierse et al. [9], similar VE estimates were derived using influenza test-negative controls and non-influenza virus positive controls.

Considering the variations of the influenza VE estimates observed by age group using non-influenza virus positive controls vs. influenza test-negative controls or pan-negative 
controls, it was observed that when stratified by age groups, particularly for the adults aged $\geq 65$ years, there was much smaller proportion of patients in non-influenza virus positive controls (Table 2). The smaller sample size might explain the variations of VE estimated using non-influenza virus positive controls compared to those using influenza test-negative controls or pan-negative controls. Influenza viruses co-circulate with other respiratory viruses in influenza season. When influenza vaccinated, the risk of a non-influenza respiratory virus infection may increase due to virus interference via a biological mechanism of temporary nonspecific immunity, resulting in a higher proportion of vaccinated patients in non-influenza virus positive controls and an overestimation of VE [6,10]. In the present study, we observed no difference in the vaccination coverage between the non-influenza virus positive controls and pan-negative controls in adults. However, when examined by age group, higher vaccination coverage $(p=0.012)$ in adults aged $18-49$ years was found in non-influenza virus positive controls vs. pan-negative controls, but this has not been seen in adults aged 50-64 years and in adults aged $\geq 65$ years (Table 4 ). Furthermore, it appeared that the differences in influenza vaccination coverage did not consistently reflect the changes of VE estimates in different age groups. Therefore, it is unlikely that influenza vaccination would be associated with the detection of non-influenza respiratory viruses. Previous studies have not shown the association between influenza vaccination and the detection of non-influenza respiratory virus [6,32]. Nevertheless, we could not rule out the possibility that influenza vaccination may affect the susceptibility to a certain or several non-influenza respiratory viruses. There is increasing evidence suggesting that there are potential virus interferences or virus interactions, which impact the dynamics of seasonal influenza $[8,33]$.

This study is subject to several limitations. First, our efforts to estimate the effect of vaccination rely on the DoD surveillance platform for data acquisition. When ascertaining vaccine status based on the self-reported questionnaire, potential non-differential misclassification could occur. In addition, the specimens collected in DoDGRS program were through routine outpatient clinical care. Before evaluating the validity of using such routinely collected data in VE analysis, the possibility of potential selection bias could not be ruled out. Second, as discussed above, there is potential impact of repeat vaccination on the VE estimates. However, the models used for VE analysis in the present study did not account for prior exposure to influenza virus antigens by repeated influenza vaccination or natural influenza virus infection in previous seasons. Similarly, there were other unmeasured confounding factors such as health status of patients. Specifically for the older adults, the age-associated changes in the immune response to influenza vaccination is influenced by the increasing level of frailty [34]. Therefore, such unmeasured factors could not be ruled out as a possible alternative explanation for the findings. Third, there were an insufficient number of patients who received high-dose influenza vaccination, thereby those patients were excluded for analysis in the present study. We were unable to compare effectiveness of high-dose and standard-dose influenza vaccine against medically attended, laboratory-confirmed influenza in adults aged $\geq 65$ years. $\mathrm{Ng}$ et al. [35] found that highdose influenza vaccine had greater antibody responses than the standard-dose influenza vaccine. In addition, studies comparing high-dose to standard-dose influenza vaccine have demonstrated increased effectiveness via high-dose influenza vaccination [36,37]. Given the limitation in the effectiveness of standard-dose influenza vaccine among the elderly, it is critical to consider alternative influenza vaccine strategies including high-dose influenza vaccine for this population [38].

\section{Conclusions}

In conclusion, the present study demonstrated moderate effectiveness of inactivated influenza vaccination in adults aged $\geq 18$ years against medically attended, laboratoryconfirmed influenza virus infection. We found the VE against influenza A(H3N2) was lower in adults aged 50-64 years than in adults aged 18-49 years. Moreover, we observed low and non-significant VE against influenza $\mathrm{A}(\mathrm{H} 1 \mathrm{~N} 1)$ pdm09 and influenza $\mathrm{A}(\mathrm{H} 3 \mathrm{~N} 2)$ in adults aged 
$\geq 65$ years. Using three different control groups, overall VE estimates against any influenza or by influenza (sub)types were consistent in adults. However, when stratified by age group, some variations of VE estimates occurred between influenza test-negative controls (or pan-negative controls) and non-influenza virus positive controls, probably due to limited number of patients included in non-influenza virus positive controls. Further research is needed to clarify the observed differences in number of patients included in different control groups. Our findings support the use of test-negative design (case vs. test-negative control) to evaluate the effectiveness of influenza vaccination in outpatient settings.

Author Contributions: Conceptualization, W.H., P.A.S., L.S.D. and A.S.R.; Data curation, W.H. and L.S.D.; Formal analysis, W.H.; Investigation, W.H., P.A.S., L.S.D. and A.S.R.; Methodology, W.H., P.A.S. and A.S.R.; Validation, W.H., P.A.S., L.S.D. and A.S.R.; Writing-original draft, W.H.; Writing-review and editing, W.H., P.A.S., L.S.D. and A.S.R. All authors have read and agreed to the published version of the manuscript.

Funding: The US Department of Defense Global Emerging Infections Surveillance Branch (DoDGEIS), Armed Forces Health Surveillance Division (AFHSD) funds the Department of Defense Global Respiratory Pathogen Surveillance (DoDGRS) Program at the US Air Force School of Aerospace Medicine at Wright-Patterson Air Force Base in Dayton, OH. JYG Innovations, LLC provided support in the form of salary for authors (W.H., L.S.D and P.A.S.).

Institutional Review Board Statement: This study was determined to be "Public Health Practice" and "Not Human Use Research", and was exempted from the review by the DoD Air Force Research Laboratory's Institutional Review Board. Regulations or rules have been followed to protect patient's identity and health information.

Informed Consent Statement: Not applicable.

Data Availability Statement: All data is presented in this article. For further information contact the corresponding author.

Acknowledgments: The authors acknowledge the contribution and support of all professionals in the DoDGRS program who are involved in the collection, collation, and reporting of surveillance data.

Conflicts of Interest: The authors declare no conflict of interest. The funders had no role in the design of the study; in the collection, analyses, or interpretation of data; in the writing of the manuscript, or in the decision to publish the results.

Disclaimer: The findings and views expressed in this article are those of the authors and do not necessarily represent the official policy or position of the USA Department of Defense.

\section{References}

1. Centers for Disease Control and Prevention. People at High Risk for Flu Complications: Flu \& People 65 Years and Older. Available online: https:/ / www.cdc.gov/flu/highrisk/65over.htm (accessed on 20 November 2021).

2. Coleman, R.; Eick-Cost, A.; Hawksworth, A.W.; Hu, Z.; Lynch, L.; Myers, C.A.; DeMarcus, L.; Federinko, S. Department of Defense end-of-season influenza vaccine effectiveness estimates for the 2017-2018 season. MSMR 2018, 25, 16-20. [PubMed]

3. Shoubaki, L.A. Department of Defense global, laboratory-based influenza surveillance program's influenza vaccine effectiveness estimates and surveillance trends for 2016-2017 influenza season. MSMR 2018, 25, 8-9. [PubMed]

4. Hu, W.; Gruner, W.E.; DeMarcus, L.S.; Thervil, J.W.; Kwaah, B.; Fries, A.C.; Sjoberg, P.A.; Robbins, A.S. Influenza surveillance trends and influenza vaccine effectiveness among Department of Defense beneficiaries during the 2019-2020 influenza season. MSMR 2021, 28, 2-8.

5. $\quad$ Kersellius, G.D.; Gruner, W.E.; Fries, A.C.; DeMarcus, L.S.; Robbins, A.S. Respiratory pathogen surveillance trends and influenza vaccine effectiveness estimates for the 2018-2019 season among Department of Defense beneficiaries. MSMR 2020, $27,17-23$.

6. Feng, S.; Fowlkes, A.L.; Steffens, A.; Finelli, L.; Cowling, B.J. Assessment of virus interference in a test-negative study of influenza vaccine effectiveness. Epidemiology 2017, 28, 514-524. [CrossRef] [PubMed]

7. Feng, S.; Cowing, B.J.; Kelly, H.; Sullivan, S.G. Estimating influenza vaccine effectiveness with the test-negative design using alternative control groups: A systematic review and meta-analysis. Am. J. Epidemiol. 2018, 187, 389-397. [CrossRef]

8. $\mathrm{Wu}, \mathrm{A}$; Mihaylova, V.T.; Landry, M.L.; Foxman, E.F. Interference between rhinovirus and influenza A virus: A clinical data analysis and experimental infection study. Lancet Microbe 2020, 1, e254-e262. [CrossRef] 
9. Pierse, N.; Kelly, H.; Thompson, M.G.; Bissielo, A.; Radke, S.; Sue Huang, Q.; Baker, M.G.; Turner, N.; on behalf of the SHIVERS Investigation Team. Influenza vaccine effectiveness for hospital and community patients using control groups with and without non-influenza respiratory viruses detected, Auckland, New Zealand 2014. Vaccine 2016, 34, 503-509. [CrossRef]

10. Van Doorn, E.; Darvishian, M.; Dijkstra, F.; Donker, G.A.; Overduin, P.; Meijer, A.; Hak, E. Influenza vaccine effectiveness estimates in the Dutch population from 2003 to 2014: The test-negative design case-control study with different control groups. Vaccine 2017, 35, 2831-2839. [CrossRef]

11. Grohskopf, L.A.; Alyanak, E.; Broder, K.R.; Blanton, L.H.; Fry, A.M.; Jernigan, D.B.; Atmar, R.L. Prevention and control of seasonal influenza with vaccines: Recommendations of the Advisory Committee on Immunization Practices-United States, 2020-2021 influenza season. MMWR Recomm. Rep. 2020, 69, 1-24. [CrossRef]

12. Hu, W.; DeMarcus, L.S.; Sjoberg, P.A.; Robbins, A.S. Inactivated influenza vaccine effectiveness among Department of Defense beneficiaries aged 6 months-17 years, 2016-2017 through 2019-2020 influenza seasons. PLoS ONE 2021, 16, e0256165. [CrossRef]

13. Belongia, E.A.; Simpson, M.D.; King, J.P.; Sundaram, M.E.; Kelley, N.S.; Osterholm, M.T.; McLean, H.Q. Variable influenza vaccine effectiveness by subtype: A systematic review and meta-analysis of test-negative design studies. Lancet Infect. Dis. 2016, 16, 942-951. [CrossRef]

14. Russell, K.; Chung, J.R.; Monto, A.S.; Martin, E.T.; Belongia, E.A.; McLean, H.Q.; Gaglani, M.; Murthy, K.; Zimmerman, R.K.; Nowalk, M.P.; et al. Influenza vaccine effectiveness in older adults compared with younger adults over five seasons. Vaccine 2018, 36, 1272-1278. [CrossRef] [PubMed]

15. Flannery, B.; Chung, J.R.; Monto, A.S.; Martin, E.T.; Belongia, E.A.; McLean, H.Q.; Gaglani, M.; Murthy, K.; Zimmerman, R.K.; Nowalk, M.P.; et al. Influenza vaccine effectiveness in the United States during the 2016-2017 season. Clin. Infect. Dis. 2019, 68, 1798-1806. [CrossRef] [PubMed]

16. Flannery, B.; Garten Kondor, R.J.; Chung, J.R.; Gaglani, M.; Reis, M.; Zimmerman, R.K.; Nowalk, M.P.; Jackson, M.L.; Jackson, L.A.; Monto, A.S.; et al. Spread of antigenically drifted influenza A(H3N2) viruses and vaccine effectiveness in the United States during the 2018-2019 season. J. Infect. Dis. 2020, 221, 8-15. [CrossRef] [PubMed]

17. Rolfes, M.A.; Flannery, B.; Chung, J.R.; O’Halloran, A.; Garg, S.; Belongia, E.A.; Gaglani, M.; Zimmerman, R.K.; Jackson, M.L.; Monto, A.S.; et al. Effects of influenza vaccination in the United States during the 2017-2018 influenza season. Clin. Infect. Dis. 2019, 69, 1845-1853. [CrossRef] [PubMed]

18. Mameli, C.; D'auria, E.; Erba, P.; Nannini, P.; Zuccotti, G.V. Influenza vaccine response: Future perspectives. Expert Opin. Biol. Ther. 2018, 18, 1-5. [CrossRef] [PubMed]

19. Crooke, S.N.; Ovsyannikova, I.G.; Poland, G.A.; Kennedy, R.B. Immunosenescence and human vaccine immune responses. Immun. Ageing 2019, 16, 25. [CrossRef] [PubMed]

20. Sullivan, S.G.; Price, O.H.; Regan, A.K. Burden, effectiveness and safety of influenza vaccines in elderly, paediatric and pregnant populations. Ther. Adv. Vaccines Immunother. 2019, 7, 1-16. [CrossRef]

21. Goodwin, K.; Viboud, C.; Simonsen, L. Antibody response to influenza vaccination in the elderly: A quantitative review. Vaccine 2006, 24, 1159-1169. [CrossRef]

22. Ferdinands, J.M.; Fry, A.M.; Reynolds, S.; Petrie, J.G.; Flannery, B.; Jackson, M.L.; Belongia, E.A. Intraseason waning of influenza vaccine protection: Evidence from the US Influenza Vaccine Effectiveness Network, 2011-2012 through 2014-2015. Clin. Infect. Dis. 2017, 64, 544-550. [PubMed]

23. Ray, G.T.; Lewis, N.; Klein, N.P.; Daley, M.F.; Wang, S.V.; Kulldorff, M.; Fireman, B. Intraseason waning of influenza vaccine effectiveness. Clin. Infect. Dis. 2019, 68, 1623-1630. [CrossRef] [PubMed]

24. Puig-Barberà, J.; Mira-Iglesias, A.; Tortajada-Girbés, M.; López-Labrador, F.X.; Librero-López, J.; Díez-Domingo, J.; CarballidoFernández, M.; Carratalá-Munuera, C.; Correcher-Medina, P.; Gil-Guillén, V.; et al. Waning protection of influenza vaccination during four influenza seasons, 2011/2012 to 2014/2015. Vaccine 2017, 35, 5799-5807. [CrossRef] [PubMed]

25. McLean, H.Q.; Thompson, M.G.; Sundaram, M.E.; Meece, J.K.; McClure, D.L.; Friedrich, T.C.; Belongia, E.A. Impact of repeated vaccination on vaccine effectiveness against influenza $\mathrm{A}(\mathrm{H} 3 \mathrm{~N} 2)$ and B during 8 seasons. Clin. Infect. Dis. 2014, 59, 1375-1385. [CrossRef]

26. Kwong, J.C.; Chung, H.; Jung, J.K.H.; Buchan, S.A.; Campigotto, A.; Campitelli, M.A.; Crowcroft, N.S.; Gubbay, J.B.; Karnauchow, T.; Katz, K.; et al. The impact of repeated vaccination using 10-year vaccination history on protection against influenza in older adults: A test-negative design study across the 2010/11 to 2015/16 influenza seasons in Ontario, Canada. Eurosurveillance 2020, 25, 1900245. [CrossRef]

27. Belongia, E.A.; Skowronski, D.M.; McLean, H.Q.; Chambers, C.; Sundaram, M.E.; De Serres, G. Repeated annual influenza vaccination and vaccine effectiveness: Review of evidence. Expert Rev. Vaccine 2017, 16, 723-736. [CrossRef]

28. Khurana, S.; Hahn, M.; Coyle, E.M.; King, L.R.; Lin, T.L.; Treanor, J.; Sant, A.; Golding, H. Repeat vaccination reduces antibody affinity maturation across different influenza vaccine platforms in humans. Nat. Commun. 2019, 10, 3338. [CrossRef]

29. Mosterín Höpping, A.; McElhaney, J.; Fonville, J.M.; Powers, D.C.; Beyer, W.E.; Smith, D.J. The confounded effects of age and exposure history in response to influenza vaccination. Vaccine 2016, 34, 540-546. [CrossRef]

30. Lu, P.J.; Hung, M.C.; O’Halloran, A.C.; Ding, H.; Srivastav, A.; Williams, W.W.; Singleton, J.A. Seasonal influenza vaccination coverage trends among adult populations, U.S., 2010-2016. Am. J. Prev. Med. 2019, 57, 458-469. [CrossRef] 
31. Nunes, B.; Machado, A.; Guiomar, R.; Pechirra, P.; Conde, P.; Cristovão, P.; Falcão, I. Estimates of 2012/13 influenza vaccine effectiveness using the case test-negative control design with different influenza negative control groups. Vaccine. 2014, 32, 4443-4449. [CrossRef]

32. Sundaram, M.E.; McClure, D.L.; VanWormer, J.J.; Friedrich, T.C.; Meece, J.K.; Belongia, E.A. Influenza vaccination is not associated with detection of noninfluenza respiratory viruses in seasonal studies of influenza vaccine effectiveness. Clin. Infect. Dis. 2013, 57, 789-793. [CrossRef] [PubMed]

33. Nickbakhsh, S.; Mair, C.; Matthews, L.; Reeve, R.; Johnson, P.C.D.; Thorburn, F.; von Wissmann, B.; Reynolds, A.; McMenamin, J.; Gunson, R.N.; et al. Virus-virus interactions impact the population dynamics of influenza and the common cold. Proc. Natl. Acad. Sci. USA 2019, 116, 27142-27150. [CrossRef] [PubMed]

34. McElhaney, J.E.; Verschoor, C.P.; Andrew, M.K.; Haynes, L.; Kuchel, G.A.; Pawelec, G. The immune response to influenza in older humans: Beyond immune senescence. Immun. Ageing 2020, 17, 10. [CrossRef] [PubMed]

35. Ng, T.W.Y.; Cowling, B.J.; Gao, H.; Thompson, M.G. Comparative immunogenicity of enhanced seasonal influenza vaccines in older adults: A systematic review and meta-analysis. J. Infect. Dis. 2019, 219, 1525-1535. [CrossRef]

36. Izurieta, H.S.; Thadani, N.; Shay, D.K.; Lu, Y.; Maurer, A.; Foppa, I.M.; Franks, R.; Pratt, D.; Forshee, R.A.; MaCurdy, T.; et al. Comparative effectiveness of high-dose versus standard-dose influenza vaccines in US residents aged 65 years and older from 2012 to 2013 using Medicare data: A retrospective cohort analysis. Lancet Infect. Dis. 2015, 15, 293-300. [CrossRef]

37. Young-Xu, Y.; Van Aalst, R.; Mahmud, S.M.; Rothman, K.J.; Snider, J.T.; Westreich, D.; Mor, V.; Gravenstein, S.; Lee, J.K.H.; Thommes, E.W.; et al. Relative vaccine effectiveness of high-dose versus standard-dose influenza vaccines among Veterans Health Administration patients. J. Infect. Dis. 2018, 217, 1718-1727. [CrossRef]

38. Whitaker, J.A.; von Itzstein, M.S.; Poland, G.A. Strategies to maximize influenza vaccine impact in older adults. Vaccine 2018, 36, 5940-5948. [CrossRef] 